\title{
Dynamic glass transition dramatically accelerates crack propagation in rubberlike solids
}

\author{
Atsushi Kubo $\odot,{ }^{1, *}{ }^{*}$ Naoyuki Sakumichi $\odot,{ }^{2, *}$ Yoshihiro Morishita, ${ }^{3}$ Ko Okumura $\odot,{ }^{4}$ Katsuhiko Tsunoda, ${ }^{5}$ \\ Kenji Urayama $\odot,{ }^{6}$ and Yoshitaka Umeno ${ }^{1}$ \\ ${ }^{1}$ Institute of Industrial Science, The University of Tokyo, Tokyo 153-8505, Japan \\ ${ }^{2}$ Department of Bioengineering, The University of Tokyo, Tokyo 113-8656, Japan \\ ${ }^{3}$ Basic Research \& Advanced Development, Bridgestone Europe NV/SA, Rome 13/15-00128, Italy \\ ${ }^{4}$ Department of Physics and Soft Matter Center, Ochanomizu University, Tokyo 112-8610, Japan \\ ${ }^{5}$ Advanced Materials Division, Bridgestone Corporation, Tokyo 187-8531, Japan \\ ${ }^{6}$ Department of Macromolecular Science and Engineering, Kyoto Institute of Technology, Kyoto 606-8585, Japan
}

(Received 25 February 2020; revised 11 May 2021; accepted 15 June 2021; published 29 July 2021)

\begin{abstract}
A crack propagating in a strained rubberlike solid exhibits an abrupt change of the propagation velocity by typically more than $10^{2}$ times at a specific threshold strain energy, which is a phenomenon called the "velocity jump." Despite its scientific and industrial significance, the mechanism of the velocity jump had been unsolved for more than 30 years. This paper gives a clear answer to the mechanism, showing dynamic glass transition at the crack tip is the true origin of the crack velocity jump. We present concerted investigations involving theoretical analysis, numerical calculation, and experiment to establish an integrated understanding of the mechanism. Our findings indicate that the velocity jump can be found in general viscoelastic materials.
\end{abstract}

DOI: 10.1103/PhysRevMaterials.5.073608

\section{INTRODUCTION}

A crack in a sufficiently stretched rubber body propagates at a velocity $V$ determined by the applied tearing energy $\Gamma$. To investigate the $V-\Gamma$ relationship, the tearing test using a "pure shear" geometry shown in Fig. 1(a) is often conducted because of the simplicity of the result: after a short transient time, a crack is expected to propagate at a constant velocity $V$ independent of the crack length, and $\Gamma$ is easily evaluated as

$$
\Gamma=W L_{0} .
$$

Here, $L_{0}$ is the height of the unstrained specimen in the tensile direction, and $W$ is the stored strain energy density obtained from the quasistatic stress $(\sigma)$-strain $\left(\varepsilon_{\text {load }}\right)$ relationship as follows:

$$
W\left(\varepsilon_{\text {load }}\right)=\int_{0}^{\varepsilon_{\text {load }}} \sigma\left(\varepsilon^{\prime}\right) d \varepsilon^{\prime} .
$$

In general, behavior of a propagating crack is not simple, as it is accompanied by several intricate phenomena such as branching [1], crack-path oscillation [2-4], supersonic rupture under ultimate strain conditions [5-7], and abrupt acceleration of crack growth [8-11]; in the present study, we discuss the abrupt acceleration of crack growth. It has been known that

\footnotetext{
*These authors contributed equally to this work.

${ }^{\dagger}$ Correspondence to be addressed: kubo@ulab.iis.u-tokyo.ac.jp
}

Published by the American Physical Society under the terms of the Creative Commons Attribution 4.0 International license. Further distribution of this work must maintain attribution to the author(s) and the published article's title, journal citation, and DOI. cracks in rubber undergo an abrupt change of the propagation velocity at a specific tearing energy $\Gamma_{\mathrm{jump}}$. This phenomenon is called the "velocity transition" $[10,11]$ or the "velocity jump" [12]. Figure 1(b) shows typical $V-\Gamma$ relationships in a previous experiment [11], where the velocity jump between the "slow" and "fast" propagation regimes is clearly observed (see movies in Supplemental Material Ref. [13]). Practically, $\Gamma_{\text {jump }}$ determines an acceptable level of external loading on rubber bodies; i.e., failure or fatigue processes can be dramatically facilitated under the loading condition $\Gamma>\Gamma_{\text {jump }}$, while for $\Gamma<\Gamma_{\text {jump }}$ crack propagation is tolerable. The mechanism of the velocity jump remained to be unveiled since Kadir and Thomas first remarked the phenomenon more than 30 years ago [8] (some may regard earlier works in 1950s [14,15] as the first reports of the velocity jump phenomenon).

Despite its importance, the velocity jump had not been sufficiently understood from the theoretical and numerical viewpoints until quite recently. Whereas a theoretical model was proposed more than 10 years ago [16,17], and some negative evidences [18] and questions [19] have further been raised.

In a recent finite element method (FEM) simulation [20], it was reported that the velocity jump can be directly reproduced with an experiment-based hyperviscoelasticity and a fracture criterion. They observed the temporal development of the stress behavior at a crack tip and explained that a nonmonotonic temporal change in stress near the crack can cause the velocity jump [Fig. 1(c)]. Under a small/large external load, the stress (or strain) at the crack tip reaches the fracture criterion after/before the local maximum point in the nonmonotonic behavior. Then, the time necessary for one element to fracture $\Delta t$ is long/short, and thus the crack propagation velocity $V\left(\propto \Delta t^{-1}\right)$ is slow/fast. This concept 
(a)

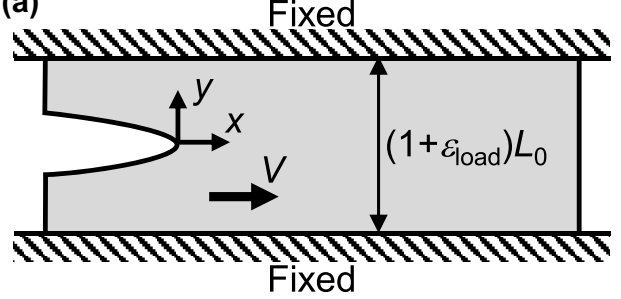

(c)

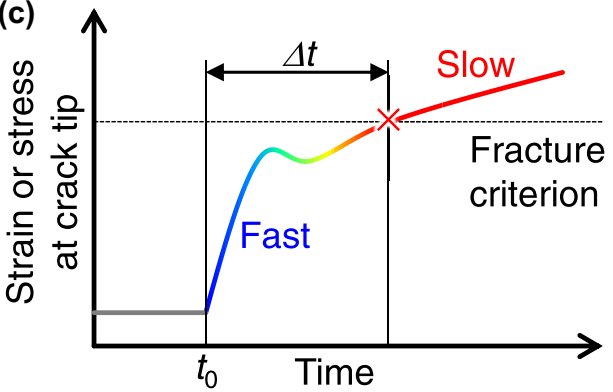

(b)

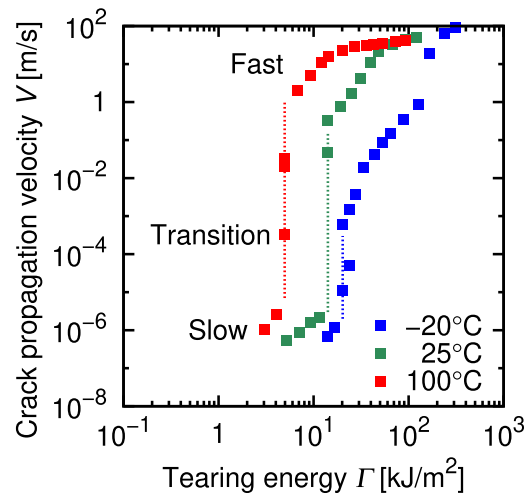

(d)

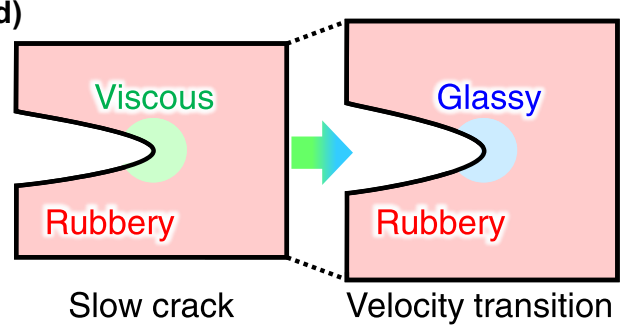

FIG. 1. Velocity jump phenomenon of crack propagation in rubber materials and hypotheses of mechanism of the jump proposed in recent researches. (a) Procedure of pure shear test. 1. The top and bottom edges of the strip specimen are clamped and stretched up to a given strain $\varepsilon_{\text {load }}$. 2. A side edge is cut to introduce an initial crack, and the crack propagation velocity $V$ is measured as a function of $\varepsilon_{\text {load }}$. 3 . After testing with various $\varepsilon_{\text {load }}$, the $V-\Gamma$ relationship is obtained via Eqs. (1) and (2). (b) Typical $V-\Gamma$ relationship in a previous experiment [11]. $V$ exhibits an abrupt jump at a specific $\Gamma$ that we call the "tearing energy at velocity jump" $\Gamma_{\text {jump }}$ (indicated by the dashed lines). Three series of data were obtained at different temperatures for an identical compound. (c) The mechanism of the velocity jump proposed by the FEM analysis [20]. At the moment of crack propagation, stress and strain rises rapidly at the crack tip (this stage is shown in blue). After a nonmonotonic behavior (in green-yellow), the strain and stress change gradually with time (in red). The time for the crack-tip element to fracture ( $\Delta t$ ) depends on at what stage the crack-tip element reaches the fracture criterion. (d) The mechanism of the velocity jump proposed by the MMCP analysis [12]. It is argued that the velocity jump appears due to a dynamic glass transition in the vicinity of the crack tip.

also explains the discontinuous nature of the velocity jump. However, the origin of such nonmonotonic mechanical behavior has remained unclear.

Meanwhile, a simple theoretical model constructed on a maximally coarse-grained lattice network with linear viscoelastic elements was proposed to give an analytical condition of the velocity jump [12]. It is argued that the velocity jump originates from a dynamic glass transition occurring at the crack tip [Fig. 1(d)]. Here, the analytical condition of the jump is obtained by taking a continuum limit along the crack propagating direction; this fact indicates that the structural discreteness along the crack propagation direction is not essential for the velocity jump. Hereafter, we call this model the minimal model for crack propagation (MMCP).

In this study, we unify the two mechanisms of the velocity jump independently proposed by the FEM simulation [20] and MMCP [12], by analytically and numerically showing that the mechanical behavior observed in the FEM simulation [20] is induced by the dynamic glass transition at the crack tip. A simplified mechanical model (step-loading model; SLM) is introduced to understand the origin of the near-tip mechanical behavior observed in the FEM simulation and to demonstrate a lucid correspondence between the FEM and MMCP analyses. In addition to theoretical and numerical analyses, we perform several crack propagation experiments to verify the mechanisms proposed by the FEM and MMCP analyses through the correlation between the tearing energy at velocity jump $\Gamma_{\text {jump }}$ and the glass transition temperature $T_{g}$. There the consistency between the experiments and the MMCP analysis is discussed with the linearity in the relationship between $\Gamma_{\text {jump }}$ and the stored energy density at break $W_{b}$. We employed several unfilled rubber compounds with material properties varied systematically for the purpose of investigating the fundamental physics in rubbers, while filled rubbers were examined in the previous studies [10,11].

The paper is organized as follows. In Sec. II, we introduce SLM with the analytical solution (Sec. II A) and explain the methodologies and procedures of the FEM simulation and the experiment (Secs. II B and II C, respectively). The derivation of the analytical solution is given in Appendix A. In addition, the stress-strain relationships and the dynamic moduli of the examined compounds are given in Appendix B to avoid digressing from the main subject. In the following two sections, we demonstrate a lucid correspondence between the FEM simulation and MMCP analyses via SLM. In Sec. III, we interpret the crack propagation mechanism in the FEM simulation from the viewpoint of the simplified mechanical model (SLM). The material parameters in SLM are given in Appendix C. In Sec. IV, we show an analytical correspondence between the MMCP and SLM based on their viscoelastic behavior. The rigorous inequalities for the relaxation times of the MMCP and SLM are shown in 


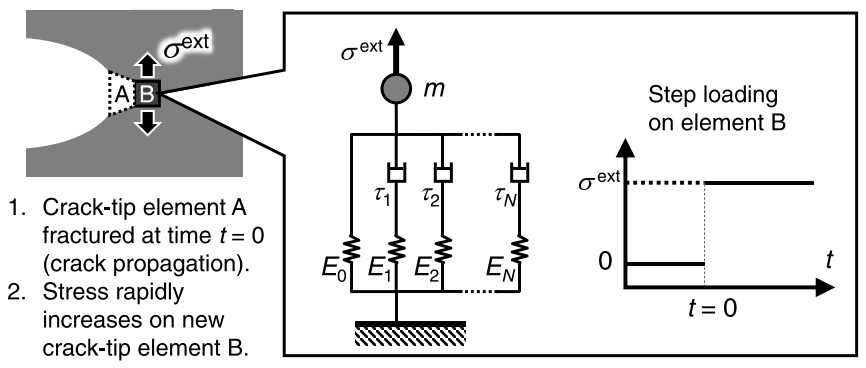

FIG. 2. Schematic illustration of a mechanical model at a crack tip (step-loading model). Fracture of the element A induces a rapid loading on the new crack-tip element B. Mechanical behavior of the element $\mathrm{B}$ in this process is modeled as a viscoelastic response to a step loading. Note that $m$ has the unit of mass per area and is dependent on the specimen height $L_{0}$.

Appendix D. We numerically compare the effective elastic modulus of the fast/slow-velocity crack propagations in Appendix E. In Sec. V, we focus on a paradoxical aspect in our discussion, which poses an apparent discord with the precedent experiments but can be resolved affirmatively. In Sec. VI, we perform several crack-propagation experiments to verify the mechanisms. In Sec. VII, we provide concluding remarks.

\section{MATERIALS AND METHODS}

\section{A. Mechanical model of crack-tip element}

We introduce a simplified mechanical model called the step-loading model (SLM) as schematically shown in Fig. 2 to understand the mechanical behavior at the crack tip observed in the previous FEM simulation [20]. Before describing the detailed formulation, we explain two major purposes of SLM.

First, SLM is applied to understand the origin of a nonmonotonic stress increase [schematically shown in Fig. 1(c)] at the crack-tip element observed in the previous FEM simulation [20]. Although the precedent FEM analysis demonstrated that the mechanism of velocity jump can be directly explained with the nonmonotonic mechanical behavior at the crack tip, its origin remained unrevealed [20]. Thus understanding the origin of the near-tip mechanical behavior is equivalent to revealing the velocity jump mechanism, and SLM is motivated to address this problem. For this purpose, our model does not need to deal with the crack propagation process itself directly, which enables a drastic simplification of the model, and is designed to reproduce the nonmonotonic mechanical behavior.

Second, SLM also aims at bridging two insights proposed in the previous studies, FEM [20] and MMCP [12]. The analysis of SLM illustrates that the mechanism of the velocity jump presented by the FEM analysis is essentially interpreted as the near-tip glass transition as was discussed in the MMCP analysis. Specifically, we clarify the relationship between the mechanical behavior (temporal developments of strain and stress) under a rapidly applied external load and the time scales of the glassy/rubbery states, by comparing the result of SLM with both the FEM and MMCP analyses. For this purpose, the model is required to cover a wide range of viscoelastic properties (the stress-relaxation curves) because the previous FEM simulation [20] dealt with a quite broad distribution of the relaxation time. Meanwhile, the model is needed to be as simple as possible for a profound mathematical analysis.

We describe the detailed formulation of SLM as shown in Fig. 2. The crack propagation process is regarded as a sequential breakage of unit elements. Breakage of an element unbalances the stress field in the crack-tip vicinity, causing a drastic change of strain and stress states. We simplify the near-tip mechanical behavior with a linear viscoelasticity, and the rapidly applied external force at the crack tip is modeled as a stepwise function without considering the geometry of the crack. It is assumed that the time between breakage of adjoining elements is sufficiently long compared to the relaxation time. Thus we obtain the mechanical model for the crack tip that consists of "a point mass connected to viscoelastic element under step loading." The temporal developments of strain $\varepsilon(t)$ and stress $\sigma(t)$ at the crack tip are derived with SLM.

To obtain the equation of motion, we represent the stress relaxation function $E(t)$ with the Prony series:

$$
E(t)=E_{0}+\sum_{i=1}^{N} E_{i} e^{-t / \tau_{i}}
$$

with the relaxed elastic modulus $E_{0}$ and the discrete spectrum $E_{i}$ related to the relaxation time $\tau_{i}(i=1, \ldots, N)$. Here, the number of relaxation times $N$, and their distribution $\tau_{i}$ are determined according to the purpose. For example, (i) to compare with the results of FEM simulations, a broad distribution of $\tau_{i}$ and thus large $N$ are necessary, and (ii) to compare with the MMCP analysis, a single relaxation time $(N=1)$.

The equation of motion for the point mass in SLM is given as follows:

$$
\begin{gathered}
m \ddot{\varepsilon}(t)=\sigma^{\mathrm{ext}} \Theta(t)-\sigma(t), \\
\sigma(t)=E_{0} \varepsilon(t)+\sum_{i=1}^{N} E_{i} \int_{0}^{t} e^{-\left(t-t^{\prime}\right) / \tau_{i}} \dot{\varepsilon}\left(t^{\prime}\right) d t^{\prime},
\end{gathered}
$$

where $\dot{\varepsilon}(t):=\frac{d \varepsilon(t)}{d t}$ and $\ddot{\varepsilon}(t):=\frac{d^{2} \varepsilon(t)}{d t^{2}}$. The constants $m$ and $\sigma^{\text {ext }}$ are the effective mass and the magnitude of external stress, respectively. The function $\Theta(t)$ is the Heaviside step function: $\Theta(t):=\left\{\begin{array}{l}1(t>0) \\ 0(t<0)\end{array}\right.$. Equation (4) can be formally solved for an arbitrary positive integer $N$ (see Appendix A), and we obtain

$$
\varepsilon(t)=\sigma^{\operatorname{ext}}\left(\frac{1}{E_{0}}+\sum_{j=1}^{N+2} C_{j} e^{-t / \hat{\tau}_{j}}\right),
$$

where $C_{j}$ and $\hat{\tau}_{j}(j=1, \ldots, N+2)$ are complex constants determined by the material parameters $m, E_{0}, E_{i}$, and $\tau_{i}(i=$ $1, \ldots, N)$. Thus $\varepsilon(t)$ is expressed as superposed exponential decays and damped oscillations, where $\hat{\tau}_{j}$ provide the relaxation times $\left(1 / \operatorname{Re}\left(1 / \hat{\tau}_{j}\right)\right)$ and frequencies $\left(\left|\operatorname{Im}\left(1 / \hat{\tau}_{j}\right)\right|\right)$. In addition, from Eqs. (4) and (6), $\sigma(t)$ is also expressed as superposed exponential decays and damped oscillations. Note that, while the eventual formulation of SLM is a simple 
TABLE I. Material properties of examined rubber samples.

\begin{tabular}{lccll}
\hline \hline & $c_{x}(\mathrm{wt} \%)$ & $T_{g}\left({ }^{\circ} \mathrm{C}\right)$ & $H_{b}(\mathrm{MPa})$ & $W_{b}(\mathrm{MPa})$ \\
\hline NBR & 1.4 & -22 & 0.880 & 0.26 \\
SBR & $1.4^{\mathrm{a}}$ & -61 & 0.0849 & 1.63 \\
BR & 1.4 & -90 & 0.0711 & 1.60 \\
\hline SBR & 0.42 & -61 & 1.07 & 0.409 \\
& 0.70 & - & 0.324 & 4.30 \\
& 0.98 & - & 0.0849 & 2.69 \\
& $1.4^{\mathrm{a}}$ & - & 0.0581 & 0.837 \\
& 2.1 & - & 0.0311 \\
\hline \hline
\end{tabular}

${ }^{\text {a Identical compound. }}$

viscoelastic model, this model fulfills the requirements for the abovementioned purposes, i.e., bridging the FEM and MMCP results.

\section{B. FEM calculation condition}

To obtain the strain and stress responses, the FEM simulations were conducted under the identical conditions to Ref. [20]. The Ogden model and the Prony series model were adopted to describe the hyperelasticity and viscoelasticity, respectively. The elastic strain energy $W$ is given by the Ogden model as follows:

$$
W=\sum_{i=1}^{N_{e}} \sum_{j=1}^{3} \frac{\mu_{i}}{a_{i}}\left(\bar{\lambda}_{j}^{a_{i}}-1\right)+K(J-1-\ln J),
$$

where $\bar{\lambda}_{j}(j=1,2,3)$ and $J$ denote the deviatoric principal stretch and relative volume change, respectively. $K, \mu_{i}$, and $a_{i}\left(i=1, \ldots, N_{e}\right)$ are material parameters. We set the number of terms for the Ogden model and the Prony series to $N_{e}=3$ and $N=12$, respectively. The material parameters were determined to reproduce the mechanical properties of an NBR sample filled with carbon black [10] (see Ref. [20] for the detail of the material parameters). The fracture criterion was given by the maximum true principal stress $(56 \mathrm{MPa})$ and the crack propagation was realized by deleting elements beyond the fracture criterion. We prepared a pure-shear specimen with the dimensions of $180 \mathrm{~mm}, 20 \mathrm{~mm}$, and $1 \mathrm{~mm}$ in the $x, y$, and $z$ directions (crack-propagation, stretching, and thickness directions), respectively. The specimen was stretched at a constant strain rate of $5 \mathrm{~s}^{-1}$ in the $y$ direction up to $\varepsilon_{\text {load }}=1.30$, where the slow-velocity crack propagation is expected. After stretching, the initial crack was introduced and the spontaneous crack propagation was observed. The size of the elements around the crack path was set to $0.50,0.19$, and $0.20 \mathrm{~mm}$ in the $x$, $y$, and $z$ directions, respectively. We focused on the temporal developments of strain $\varepsilon(t)$ and stress $\sigma(t)$ at an element on the crack path [Fig. 4(b), discussed in detail in Sec. III].

\section{Experiment}

Preparation and characterization of samples. We adopted three types of unfilled rubber vulcanizates for the crack propagation tests, i.e., acrylonitrile butadiene rubber (NBR), styrene butadiene rubber (SBR), and butadiene rubber (BR), whose key material properties are listed in Table I. We prepared two series of compounds: (a) NBR, SBR, and BR samples with an equal sulfur cross-linker concentration $c_{x}=1.4 \mathrm{wt} \%$ for investigation of the effect of $T_{g}$ on $\Gamma_{\text {jump }}$; (b) six SBR samples with various $c_{x}=0.42-2.8 \mathrm{wt} \%$ (one of them is identical to a sample in (a), i.e., $c_{x}=1.4 \mathrm{wt} \%$ ) for investigation of the effect of $W_{b}$ on $\Gamma_{\text {jump. }}$. Characterization of samples were conducted with uncracked specimens independently of crack propagation tests, in a way similar to the previous experiments $[10,11]$. The energy density at break $W_{b}$ was evaluated from the stress-strain relationship $\sigma\left(\varepsilon_{\text {load }}\right)$ of uncracked specimens and the strain at break $\varepsilon_{b}$ with Eq. (2), which is schematically shown as the sum of red and blue areas in Fig. 3. Note that $W_{b}$ is not a fracture parameter but the quasistatic strain energy density that a given rubber is able to sustain. Hysteresis loss at break $H_{b}$ was obtained from the difference in the stressstrain relationships during loading and unloading processes (shown as the red area in Fig. 3). The uni-axial tensile test was carried out 8 times for each material to obtain $\sigma\left(\varepsilon_{\text {load }}\right)$ and $\varepsilon_{b}$. The dynamic viscoelastic behavior was evaluated by

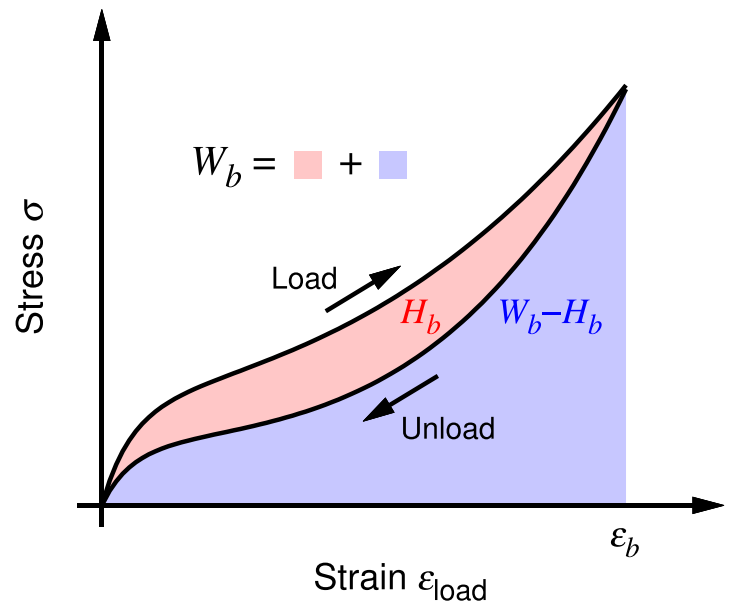

FIG. 3. Schematic illustration of typical stress-strain relationship of uncracked rubber specimen under loading and unloading conditions. The area of stress-strain curve integrated up to the strain at break $\varepsilon_{b}$ indicates the strain energy density at break [i.e., Eq. (2) with $\varepsilon_{\text {load }}=\varepsilon_{b}$ ]; the sum of red and blue areas is the energy density applied during the loading process $\left(W_{b}\right)$, the blue area is the energy released during the unloading process, and the red area is the hysteresis loss during the loading cycle $\left(H_{b}\right)$. 



FIG. 4. Mechanical response obtained by SLM and FEM analyses. (a) Step-loading response obtained by SLM analysis: (top) strain response and (bottom) stress response. The normalization factors for strain and stress are defined as $\varepsilon_{\infty}:=\sigma^{\text {ext }} / E_{0}$ and $\sigma_{\infty}:=\sigma^{\text {ext }}$, respectively. Three representative regimes are indicated in different colors according to the explanation in Ref [20]; the blue/green (I/II) boundary is determined by the local maximum point of strain, and the green/red (II/III) boundary is determined by the time at that the strain reaches again to the level of the local maximum point; i.e., the strain values at the both boundaries are equal. (b) Mechanical response at a crack tip obtained with FEM simulation under the condition identical to that in Ref. [20]: (top) maximum nominal principal strain; (bottom) maximum true principal stress.

applying an oscillatory shear deformation at $0.4 \%$ strain to a cylindrical sample. The stress-strain relationships and the dynamic moduli are given in Appendix B.

Crack propagation test. Crack propagation tests were performed with the pure shear geometry as shown in Fig. 1(a), in a way similar to the previous experiments [10,11]. We prepared the unstrained specimens of NBR, SBR, and BR with the dimensions of $180 \times 20 \times 1 \mathrm{~mm}$ in $x \times y \times z$ directions (identical to the FEM simulation). Specimens were clamped and stretched [Fig. 1(a)] up to a given strain $\varepsilon_{\text {load }}$ at a constant stretch speed $1 \mathrm{~mm} / \mathrm{s}$. After stretching the specimen, an edge of the specimen was cut to introduce an initial crack. The temporal development of crack length $c(t)$ was measured with a high-speed camera to evaluate the crack propagation velocity $V=d c / d t$. The tearing energy $\Gamma$ was calculated from Eqs. (1) and (2). Since a crack is expected to propagate at a constant velocity under the pure-shear condition, each test produces one data point in the $V-\Gamma$ relationship. Repeating this procedure with various $\varepsilon_{\text {load }}$, we obtain the whole $V-\Gamma$ relationship. All the crack propagation tests were carried out at $T=298 \mathrm{~K}$.

\section{CORRESPONDENCE BETWEEN SLM AND FEM ANALYSES}

We present that the equation of motion for SLM (4) can reproduce the crack-tip behavior observed in the FEM simulation. Here, the parameters in the Prony series (3) were determined based on an experimental stress relaxation function $E(t)$ with $N=25$ and $\tau_{i}=10^{-10}-10^{2}$ s (see Appendix C). The strain and stress behaviors obtained are shown in Fig. 4(a). For comparison Fig. 4(b) shows the mechanical response at the crack tip in the FEM simulation under the condition identical to that employed in Ref. [20]. In the strain response in SLM [Fig. 4(a) top], the following three regimes are observed: (I) a sharp increase right after the step loading; (II) a nonmonotonic behavior (accompanied with a local maximum point); and (III) a moderate increase to the fully relaxed state.

This three-regime behavior resembles the strain behavior at the crack tip obtained by the FEM simulation [Fig. 4(b) top]. In addition, the time scale of crossover from regime I to II is $\sim 0.01 \mathrm{~ms}$ both for the FEM simulation and the SLM analysis. These agreements illustrate that the nonmonotonic mechanical behavior at the crack tip (regime II) can be understood as a typical viscoelastic response to a rapid loading. Note that the SLM analysis [Fig. 4(a) bottom] does not reproduce the moderate increase of stress in the regime III in the FEM simulation [Fig. 4(b) bottom], presumably due to our oversimplified assumptions; e.g., we omitted the effects of nonlinear elasticity, finite deformation in crack-tip vicinity, etc.

The steep rise in strain (regime I) and the gentle rise (regime III) correspond to relatively small and large relaxation times in $\hat{\tau}_{j}$, respectively. As shown below, the regimes I and III can be respectively regarded as the glassy and rubbery states, which is consistent with the MMCP analysis.

\section{CORRESPONDENCE BETWEEN SLM AND MMCP ANALYSES}

We analytically show that regimes I and III in Fig. 4(a) correspond to the glassy and rubbery states, respectively, using SLM with a single time constant $\tau:=\tau_{1}$, i.e., Eq. (3) with $N=1$ (the so-called Zener model). As shown in Fig. 5(a), the Zener model is a minimal model that exhibits rubbery, viscous, and glassy behaviors, depending on the strain rate. To characterize the Zener model, it is convenient to define 

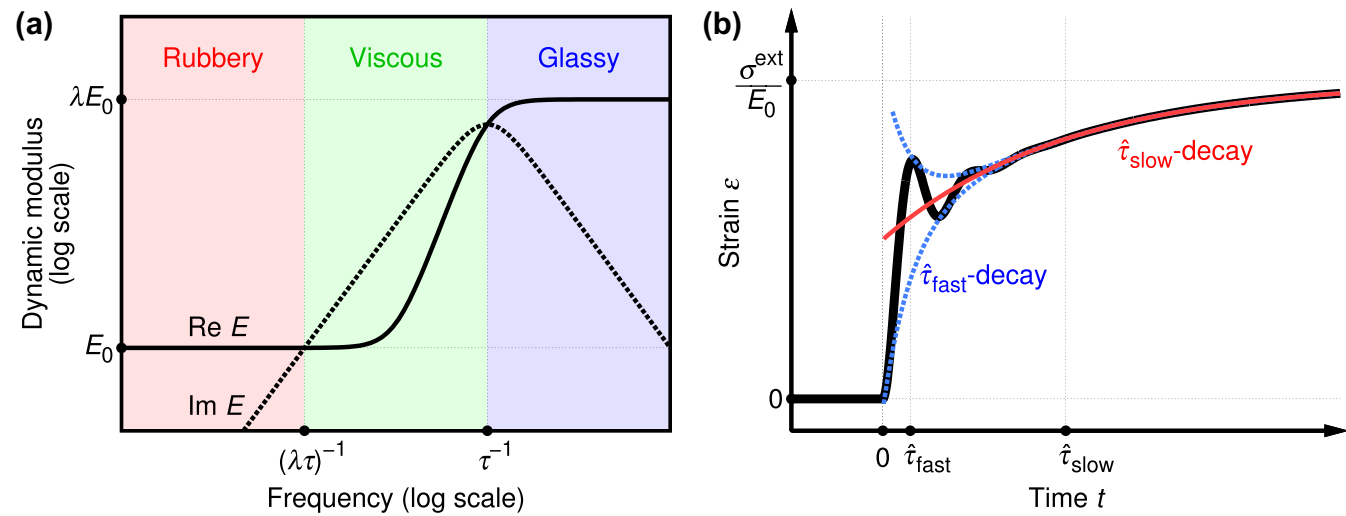

FIG. 5. Viscoelastic behavior of a Zener element and mechanical response of SLM. (a) Dynamic modulus $E$ of a Zener element as a function of frequency. $E_{0}$ and $\lambda E_{0}$ correspond to the elastic moduli at the rubbery and glassy states, respectively. (b) Schematic illustration of strain response in SLM (black line). This behavior is characterized by three parameters, i.e., $\hat{\tau}_{\text {slow }}, \hat{\tau}_{\text {fast }}$, and $\omega$. The contributions from $\hat{\tau}_{\text {slow }}$ and $\hat{\tau}_{\text {fast }}$ are represented by a solid red line and dashed blue lines, respectively.

the ratio of elastic constants of glassy and rubbery states $\lambda:=$ $1+E_{1} / E_{0}$. Parameters $E_{0}$ and $\lambda E_{0}$ represent elastic moduli of rubbery and glassy states, respectively. Also, $\tau$ and $\lambda \tau$ are respectively the relaxation time at the glassy and rubbery states.

The corresponding strain response in Eq. (6) with $N=1$ is $\epsilon(t)=\sigma^{\operatorname{ext}}\left(\frac{1}{E_{0}}+\sum_{j=1}^{3} C_{j} e^{-t / \hat{\tau}_{j}}\right)$. We can show that, for sufficiently small $m,\left\{\hat{\tau}_{j}\right\}_{j=1,2,3}$ consists of a positive real number $\hat{\tau}_{1}$ and a pair of complex conjugates $\hat{\tau}_{2}, \hat{\tau}_{3}$ (see Appendix D). Then, the strain response is described as a superposition of an exponential decay and a damped oscillation:

$$
\epsilon(t)=\sigma^{\operatorname{ext}}\left[\frac{1}{E_{0}}+C_{1} e^{-t / \hat{\tau}_{\text {slow }}}+C_{0} e^{-t / \hat{\tau}_{\text {fast }}} \sin (\omega t+\theta)\right],
$$

where the three real constants, $C_{0}, C_{1}$, and $\theta$ are determined by initial conditions [Eq. (A1) in Appendix A]. Here, $\hat{\tau}_{\text {slow }}:=$ $\hat{\tau}_{1}, 1 / \hat{\tau}_{\text {fast }}:=\operatorname{Re}\left(1 / \hat{\tau}_{2}\right)\left(=\operatorname{Re}\left(1 / \hat{\tau}_{3}\right)\right)$, and $\omega:=\operatorname{Im}\left(1 / \hat{\tau}_{2}\right)(=$ $\left.-\operatorname{Im}\left(1 / \hat{\tau}_{3}\right)\right)$ are positive real numbers, dependent on material parameters $(\tau, \lambda$, and $m$ ). As schematically shown in Fig. 5(b), $\hat{\tau}_{\text {slow }}$ and $\hat{\tau}_{\text {fast }}$ express the relaxation times at long and short times, respectively, and $\omega$ gives the angular frequency of the damped oscillation.

When $\lambda \gg 1$ ( $\lambda \simeq 10^{3}$ for typical rubbers), we obtain

$$
\begin{aligned}
& \hat{\tau}_{\text {fast }} \simeq 2 \tau, \\
& \hat{\tau}_{\text {slow }} \simeq \lambda \tau
\end{aligned}
$$

(see Appendix D for the derivation). Since $\tau$ and $\lambda \tau$ are respectively the relaxation time in the glassy and rubbery states in the Zener model, regimes I and III in Fig. 4(a) correspond to the glassy and rubbery states, respectively [besides the factor of 2 in Eq. (9)].

We clarify the relationship between the formulations of SLM and MMCP. While the velocity jump was reproduced in MMCP by taking the continuum limit along the crack propagating direction [12], below we consider the discrete model before taking the continuum limit for the convenience of discussion. We note that whether or not to take the continuum limit along the crack propagating direction is not essential for the occurrence of the velocity jump because the velocity jump occurs in both discrete [20] and continuum [12] models (Instead, the discreteness in the direction perpendicular to the crack path is essential.) The equation of motion in the direction perpendicular to the crack path in MMCP (Eq. (12) in Ref. [12]) is given by

$$
0=-\sigma_{n}(t)+E_{0} \varepsilon_{\text {load }}-\sigma_{n}^{\text {shear }}(t),
$$

where $n=1,2, \ldots$ label Zener models (short springs) horizontally aligned along the crack propagating direction in front of the crack. The viscoelastic normal stress $\sigma_{n}(t)$ is given by Eq. (5) together with $N=1, \epsilon(t)=\epsilon_{n}(t)$, and $\dot{\epsilon}\left(t^{\prime}\right)=\dot{\epsilon}_{n}\left(t^{\prime}\right)$; $\varepsilon_{\text {load }}$ is a given real constant; the shear stress between the neighboring elements is given by

$$
\sigma_{n}^{\text {shear }}(t):=\frac{\mu}{2}\left(1-\frac{l}{L}\right)\left[\epsilon_{n+1}(t)-2 \epsilon_{n}(t)+\epsilon_{n-1}(t)\right],
$$

where $\mu, L$, and $l$ are the effective shear modulus, the height of the specimen under zero strain, and the lattice spacing (corresponding to the natural length of the short springs) in the direction perpendicular to the crack path, respectively. (The exact definition of $\mu, L$, and $l$ are given in Ref. [12].) Then, the equation of motion in MMCP (11) can be converted to that in SLM (4) with $N=1$, only by introducing the step-loading term and the mass term instead of the shear term $\sigma_{n}^{\text {shear }}(t)$. Therefore time constants [Eqs. (9) and (10)] in SLM correspond to those in MMCP.

We summarize the above analyses. The mechanism of the velocity jump proposed by the MMCP and the FEM simulation were bridged through SLM as follows. First, we related SLM to FEM; according to the comparison in Fig. 4, the SLM analysis well reproduces the mechanical behavior at the crack tip in the FEM simulation. Second, we related SLM to MMCP through an analytical correspondence of the time constants between MMCP and SLM. Finally, SLM connects the fast/slow-velocity cracks with the glassy/rubbery states, which shows the consistency between FEM and MMCP.

Note that the above discussion for the Zener model (i.e., $N=1$ ) can be extended to the case with arbitrary $N$. We can show a numerical correspondence between the fast/slow-velocity crack propagations and the glassy/rubbery states by comparing the effective elastic modulus $E_{\text {eff }}(t):=$ 

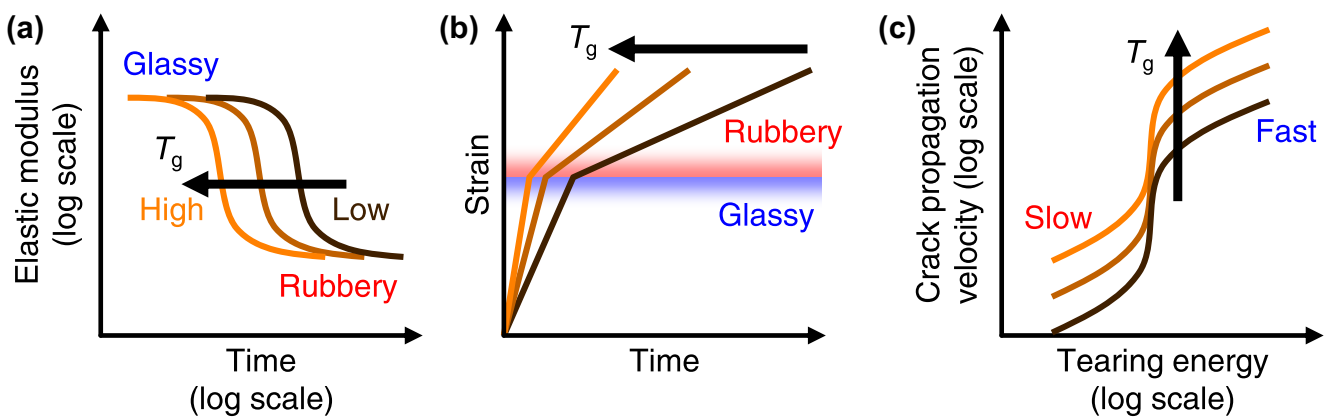

FIG. 6. Schematic illustrations for deduction of $T_{g}$-dependence in SLM. (a) Stress relaxation functions with various $T_{g}$. (b) Corresponding strain behaviors in SLM (the glass-rubber transition behavior is simplified and exaggerated for clarity). (c) Expected relationships between the tearing energy and crack propagation velocity, where the tearing energy at velocity jump is not affected by $T_{g}$.

$\sigma^{\text {ext }} / \varepsilon(t)$ with $E(t)$. The result of the comparison is shown in Appendix E.

\section{PARADOXICALITY IN $\boldsymbol{\Gamma}_{\text {jump }}-\boldsymbol{T}_{g}$ RELATIONSHIP}

The statement that the velocity jump originates from the glass transition in the crack-tip vicinity may lead one to the following erroneous deduction: "Glass transition occurs more easily for a material with higher $T_{g}$ because of a smaller gap between $T_{g}$ and the experimental room temperature $T\left(>T_{g}\right.$ ). Therefore such a material has a smaller $\Gamma_{\text {jump }}$ and a negative correlation is expected between $\Gamma_{\text {jump }}$ and $T_{g}$." In what follows we explain why this deduction is not correct.

As shown in Eq. (8) in Ref. [12], we can obtain the analytic formula for $\Gamma_{\text {jump }}$ in MMCP as

$$
\Gamma_{\text {jump }}=l \lambda W_{b},
$$

where $\lambda:=1+E_{1} / E_{0}$ is defined in Sec. IV, and $W_{b}=W\left(\varepsilon_{b}\right)$ is calculated from Eq. (2) with the strain at break $\varepsilon_{b}$. Since the exact definition of $l$ and a mathematical proof of Eq. (13) have been explained in Ref. [12], we only briefly describe the results here. In MMCP, $l$ denotes the lattice spacing corresponding to the natural length of the portion of the material that is to be broken by crack propagation. We can consider $l$ as a microscopic length scale on which the continuum description is invalidated. Thus $l$ is the largest length scale among the scales such as the cross-link distance, the size of filler particles, the filler-particle distance, and the length scale of the inhomogeneous structure in the sample. It is evident that $\Gamma_{\text {jump }}$ is not an explicit function of $T_{g}$ and the effect of $T_{g}$ is presumably introduced via $l, \lambda$ and especially $W_{b}$.

The similar deduction can proceed for the strain behavior in SLM, suggesting an indirect effect of $T_{g}$ on $\Gamma_{\text {jump }}$. We assume $m=0$ for simplicity. Although the temperature-related effects were omitted in the present FEM analyses as well as the previous analysis [20], one can deduce how the glass transition temperature $T_{g}$ affects the strain behavior in SLM by the time-temperature equivalence. That is, the effect of $T_{g}$ can be considered through the shift of the relaxation times $\tau$ instead of direct introduction of the $T_{g}$-effect. If we shift $\tau_{i}$ to $a \tau_{i}$ with a coefficient (shift factor) $a$ to realize the effect of $T_{g}$ as shown in Fig. 6(a), the shift is equivalent to changing the unit of time by the factor of $1 / a$. Then, the strain response of SLM is accordingly shifted along the horizontal axis (time) by $a$ times. The whole curve shape however does not substantially change, nor is the strain level to cause the velocity jump affected [see Fig. 6(b)]. Therefore the tearing energy at velocity jump $\Gamma_{\text {jump }}$ is not affected by such an operation, whereas a shift of time scale may move the entire $\Gamma-V$ curve uniformly in parallel along the ordinate (crack propagation velocity), as shown in Fig. 6(c). Note that an effect of $T_{g}$ is possible if $m$ is sufficiently large because the inertial and viscous terms are not equally dependent on $T_{g} ; m$ is independent of $T_{g}$ while $\tau$ is influenced.

By using empirical relations, we can show that there is a positive correlation between $T_{g}$ and $\Gamma_{\text {jump }}$, the reason for which is discussed below. Here, we relate $\Gamma_{\text {jump }}$ to $T_{g}$ via the following diagram:



Here, $c_{x}$ and $H_{b}$ denote the cross-linker concentration and the hysteresis loss at break, respectively. The arrows (a), (b), (c), and (d) indicate the correlations between the physical quantities. Figure 7 demonstrate the $c_{x}-H_{b}-W_{b}$ correlations for the SBR compounds with various $c_{x}$ and the $c_{x}-H_{b}-W_{b}$ correlations for the three compounds (NBR, SBR, and BR) with equal $c_{x}$, respectively. A negative $c_{x}-H_{b}$ correlation and a positive $T_{g}-H_{b}$ correlation have been empirically known for (a), and in this study the $c_{x}$ - and $T_{g}$-dependences of $H_{b}$ were examined for several rubber compounds. Correlation (b) was investigated by previous experiments and found to be expressed as a power law [21-23]:

$$
W_{b}=K H_{b}^{2 / 3} \text {, }
$$

where $K$ is a material-dependent parameter [23]. According to tensile tests for several rubber compounds [22,23], $W_{b}$ has positive and negative correlations with $T_{g}$ and $T$, respectively [i.e., correlation (c)]. Correlation (d) is derived from MMCP as Eq. (13), a linear relationship [12]. Thus correlations (c) and (d) result in a positive correlation between $\Gamma_{\text {jump }}$ and $T_{g}$ (and a negative correlation between $\Gamma_{\text {jump }}$ and $T$ ). Note that these correlations were derived from MMCP analysis with the experiments for uncracked body, i.e., without crack propagation experiments. Below, we perform crack propagation experiments, and a positive correlation between $\Gamma_{\text {jump }}$ and $T_{g}$ is experimentally confirmed by comparing three different rubber materials (i.e., NBR, SBR, and BR) under an identical 

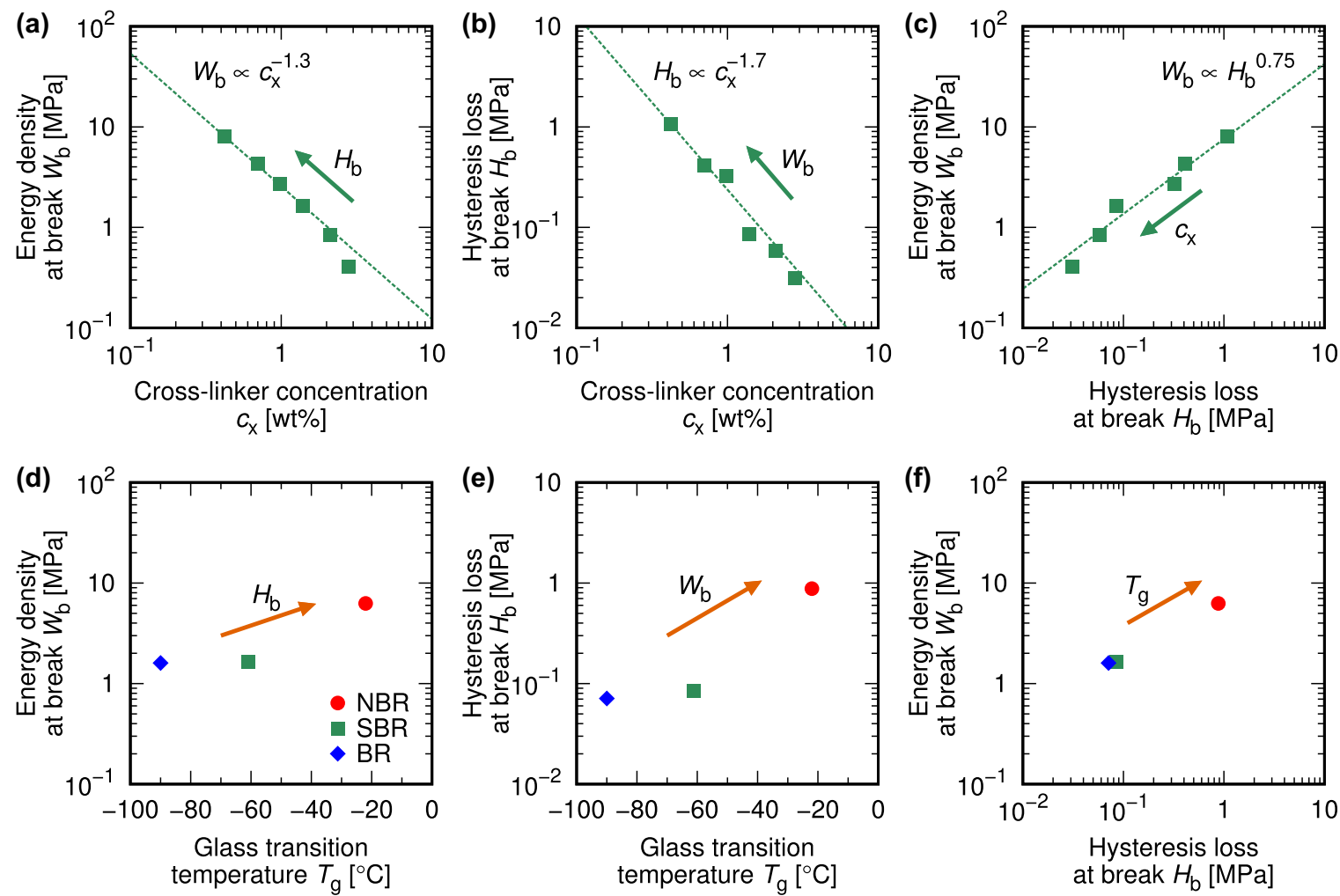

FIG. 7. Correlations between $c_{x}, T_{g}, H_{b}$, and $W_{b}$. [(a)-(c)] Correlations between $c_{x}, H_{b}$, and $W_{b}$ for SBR's with various $c_{x}$ obtained by experiment; (a) $W_{b}-c_{x}$, (b) $H_{b}-c_{x}$, and (c) $W_{b}-H_{b}$ relationships. The dashed lines indicate the power-law approximations of the correlations. [(d)-(f)] Correlations between $T_{g}, H_{b}$, and $W_{b}$ for NBR, SBR, and BR compounds with equal $c_{x}$ obtained by experiment; (d) $W_{b}-T_{g}$, (e) $H_{b}-T_{g}$, and (f) $W_{b}-H_{b}$ relationships.

condition of the experimental room temperature, cross-linker concentration.

\section{VALIDATION OF THEORETICAL PREDICTION BY EXPERIMENT}

We perform a series of crack propagation experiments to validate the insight from FEM, MMCP and SLM analyses. Instead of challenging work to observe the glass transition at the crack tip, we compare the $\Gamma_{\text {jump }}-W_{b}$ relationships obtained by the experiments and the theoretical prediction by MMCP [12]. The crack-propagation experiments demonstrate the following three facts. (1) The relationship between $\Gamma_{\text {jump }}$ and $T_{g}$ shows a positive correlation (and a negative correlation between $\Gamma_{\text {jump }}$ and $T$ ). (2) The effect of $T_{g}$ and $T$ on $\Gamma_{\text {jump }}$ appears via the material parameters $l, \lambda$, and particularly $W_{b}$. In other words, $\Gamma_{\text {jump }}$ is not given as an explicit function of $T_{g}$ and $T$, but implicitly affected via $W_{b}$. (3) If $l$ and $\lambda$ are dependent on $T$ only weakly, $\Gamma_{\text {jump }}$ is proportional to $W_{b}$.

Provided that $\lambda$ and $l$ are constant independently of the rubber compounds, we expect $\Gamma_{\text {jump }} \propto W_{b}$ even if $T_{g}, T$, and $c_{x}$ are varied. Since there is a previous experiment that examined the effect of temperature on $\Gamma_{\text {jump }}$ [11], we investigate the $\Gamma_{\text {jump }}-W_{b}$ relationship at a constant temperature dealing with $T_{g}$ and $W_{b}$ as variables. First, to investigate the effect of $T_{g}$, three different rubber compounds were examined, i.e., acrylonitrile butadiene rubber (NBR), styrene butadiene rubber (SBR) and butadiene rubber (BR), all of which are unfilled and cross-linked with an equal cross-linker concentration $c_{x}$.
For three compounds, the elastic moduli at the glassy and rubbery states are approximately equal (i.e., $\lambda \simeq$ const.) but relaxation times and thus $T_{g}$ 's are different. Second, to investigate the effect of $W_{b}$ on $\Gamma_{\text {jump }}$ independently of $T_{g}$, we examined six SBR compounds with various $c_{x}$, viscoelastic behavior of which are substantially equal (see Appendix B for further details of the material properties).

Figure 8 shows the $V-\Gamma$ relationships at $T=298 \mathrm{~K}$ for three rubber compounds with equal $c_{x}$ and those for SBR with various $c_{x}$. In all the cases, the velocity jump was clearly observed. Figure 9 shows the $\Gamma_{\text {jump }}-W_{b}$ relationship obtained from the present and previous [11] experiments. This relationship shows a clear linearity, regardless of $T_{g}, T$ or $c_{x}$, which suggests that the effects of $T_{g}$ and $T$ on $\Gamma_{\text {jump }}$ are actualized as influences on $W_{b}$. The magnitude of $\Gamma_{\text {jump }}$ was in the order as $\mathrm{NBR}>\mathrm{SBR}>\mathrm{BR}$, which is in accordance with that of $T_{g}$. This result is qualitatively consistent with the theoretical prediction by MMCP (13) with experimental results for uncracked bodies [22,23]. In addition, the previous experiment reports a negative correlation between $\Gamma_{\text {jump }}$ and $T$ [11], which is also consistent with the MMCP prediction. The apparent negative correlation between $\Gamma_{\text {jump }}$ and $T$ in the previous study is derived from the temperature effect on $W_{b}$.

The above remarks demonstrate the consistency between the MMCP predictions and the experiments by confirming linearity in the $\Gamma_{\text {jump }}-W_{b}$ relationship. Besides the present results, some previous experiments imply the role of the glass transition in the velocity jump. According to the experimental observation [8], "the fracture surfaces are smooth and 'glassy' 

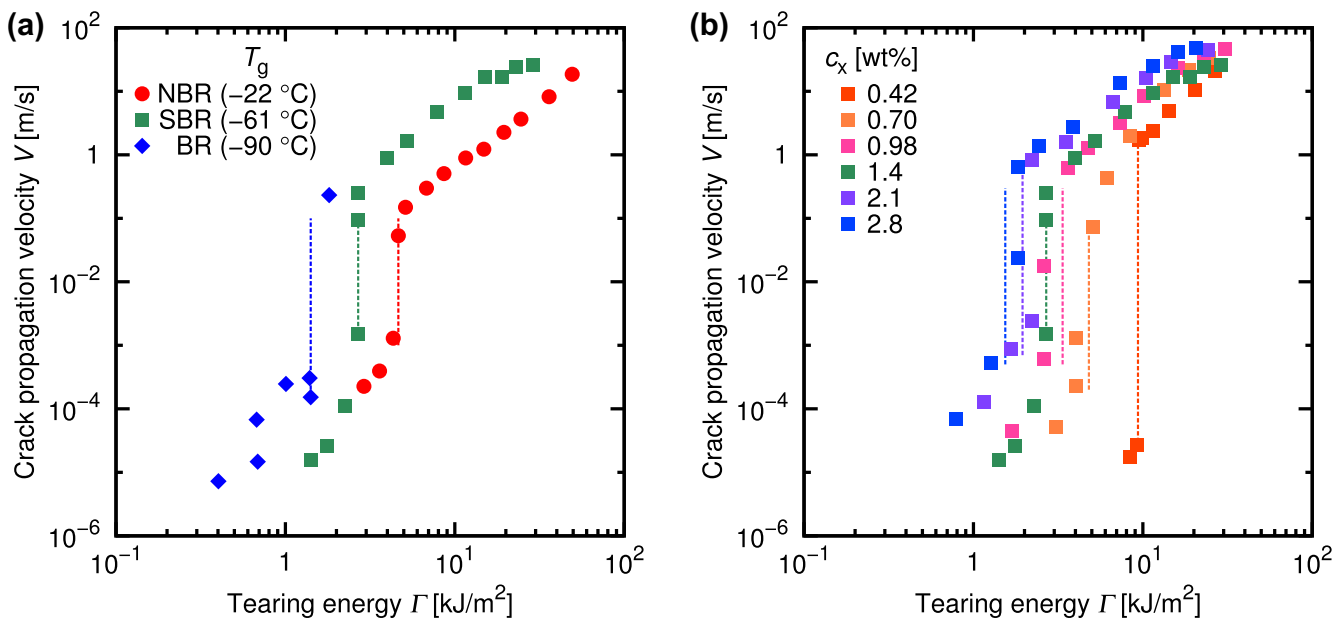

FIG. 8. Crack propagation velocity $V$ as a function of tearing energy $\Gamma$. The relationships were obtained at $T=298 \mathrm{~K}$ with (a) three different rubbers (NBR, SBR, BR; all cross-linked and unfilled) with identical cross-linker concentrations $c_{x}=1.4$ wt $\%$ and (b) SBR with various $c_{x}$. The data for SBR with $c_{x}=1.4 \mathrm{wt} \%$ are shown in both (a) and (b), indicated by green squares. The vertical lines indicate the velocity jump (guide for eyes).

in appearance" at the fast crack propagation. Similar remarks are found in other experimental observations of fracture surfaces [24].

Note that the MMCP prediction for the $\Gamma_{\text {jump }}-W_{b}$ relationship is valid only for approximately linear elastic materials;



FIG. 9. Tearing energy at velocity jump $\Gamma_{\text {jump }}$ as a function of energy density at break $W_{b}$ obtained by experiments. The previous result is adopted from Ref. [11], in which data were obtained at various temperatures for an identical compound [cross-linked SBR with silica filler, shown in Fig. 1(b) in this paper]. All data in this work were obtained with unfilled compounds at $T=298 \mathrm{~K}$ [Figs. 8(a) and 8(b)]. The orange circles indicate three different substances (NBR, SBR, and BR) with identical $c_{x}$ but different $T_{g}$. The dashed line shows a result of linear fitting to the data. The error bars indicate the standard deviation of $W_{b}$ examined with 8 specimens for each compound. otherwise, e.g., for filled elastomers of practical use, the $\Gamma_{\text {jump }}-W_{b}$ relationship is deviated from linearity as discussed in Ref. [11]. For such compounds, it is inevitable to consider the effect of nonlinear elasticity, and a linear master curve for the $\Gamma_{\text {jump }}-W_{b}$ relationship is obtained [11] after compensating the effect of nonlinearity by the weakly nonlinear theory of dynamic fracture [25-27].

\section{CONCLUDING REMARKS}

The velocity jump of crack propagation in rubber was investigated with theoretical modeling, numerical simulation, and experiment to explain the velocity jump phenomenon as the dynamic glass transition at the crack tip. We gave a unified understanding of the mechanisms of the velocity jump independently proposed by the previous FEM simulation [20] and the MMCP analysis [12]. The mechanical model called SLM was developed to understand the near-tip mechanical behavior in the FEM simulation. As a result, it was revealed that the mechanism of the velocity jump proposed by the FEM analysis is derived from the dynamic glass transition at the crack tip, which is consistent with the MMCP analyses. MMCP predicts a proportional $\Gamma_{\text {jump }}-W_{b}$ relationship if the material parameters $l$ and $\lambda$ are constant. It is interesting that $\Gamma_{\text {jump }}$ has no direct relevance to $T_{g}$ while MMCP attributes the velocity jump to the dynamic glass transition in the crack-tip vicinity; the effect of $T_{g}$ on $\Gamma_{\text {jump }}$ appears mainly via that on $W_{b}$.

The FEM simulation and the MMCP analysis both focus on the viscoelasticity of materials and omit the effects of temperature, plasticity, etc. Thus we demonstrated that the temperature effect is not necessarily required to reproduce the velocity jump, while the previous theory [16,17] assumes a unrealistic rise in temperature $(\sim 1000 \mathrm{~K})$ near the crack tip. Indeed, only a modest increase in the temperature was observed in an experiment [18] (further criticisms are found in Ref. [19]). Since both the FEM and MMCP analyses adopt fracture criteria based on strain or stress at break instead of fracture parameters such as toughness, their relation to 
conventional fracture mechanics is somewhat unclear. However, they may be properly connected, for example, by converting strength parameters and characteristic length into fracture parameters. A full-scale discussion about their relationship remains as our future work.

As discussed above, the previous work [11] suggests a linear $\Gamma_{\text {jump }}-W_{b}$ relationship even for the filled rubber compounds after the effect of nonlinear elasticity is properly compensated by the weakly nonlinear theory [25-27]. It is therefore worth noting that the simple assumptions in MMCP do not mar the nature of the velocity jump phenomenon despite the importance of the nonlinear elasticity and broadly distributed relaxation time in rubbers $[10,19,28]$; a simple material model is useful for explaining the qualitative aspect of the velocity jump.

Lastly, we note that MMCP does not assume any special material properties but an ordinary viscoelasticity, and we therefore consider that the velocity jump may be found in general viscoelastic materials, not limited to rubberlike solids. Indeed, very recent experiments [29-32] have reported the velocity jump phenomenon occurring on various types of polymer materials.

\section{ACKNOWLEDGMENTS}

This research was funded by ImPACT Program of Council for Science, Technology and Innovation (Cabinet Office, Government of Japan). This work was supported by JSPS KAKENHI Grant No. JP19K14839 and JP19K14672.

A.K. designed and performed the FEM simulations. N.S. conceived and solved the mathematical model. Y.M. designed and performed the experiments. Y.U., K.O., K.T., and K.U. designed and supervised the entire research. A.K. and N.S. wrote the manuscript. Y.M. and K.T. prepared supplementary movies. All authors discussed the results and commented on the manuscript.

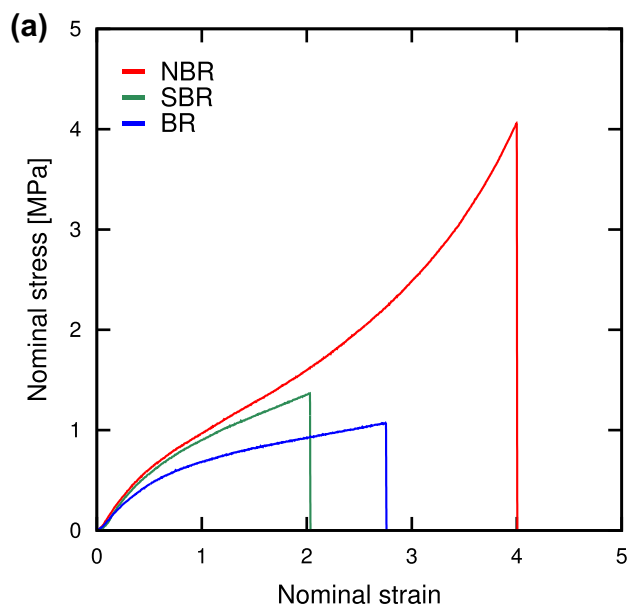

\section{APPENDIX A: SOLUTION OF STEP-LOADING MODEL}

We derive Eq. (6) as the solution of the equation of motion for SLM (4) for general $N$. Applying the Laplace transform to Eq. (4) under the initial conditions:

$$
\varepsilon(0)=0 \quad \text { and } \quad \dot{\varepsilon}(0)=0,
$$

we obtain

$$
m s^{2} Y(s)+E_{0} Y(s)+\sum_{i=1}^{N} \frac{E_{i} s Y(s)}{s+1 / \tau_{i}}=\frac{\sigma^{\text {ext }}}{s},
$$

where $Y(s)=\int_{0}^{\infty} \varepsilon(t) e^{-s t} d t$ is the Laplace transform of $\varepsilon(t)$. Solving Eq. (A2) for $Y(s)$, we obtain $Y(s)=\sigma^{\text {ext }} /(s f(s))$, where

$$
f(s):=m s^{2}+E_{0}+\sum_{i=1}^{N} \frac{E_{i} s}{s+1 / \tau_{i}} .
$$

$Y(s)$ can be formally transformed to

$$
Y(s)=\sigma^{\mathrm{ext}}\left(\frac{1}{E_{0} s}+\sum_{j=1}^{N+2} \frac{C_{j}}{s-\alpha_{j}}\right),
$$

where $\alpha_{j} \in \mathbb{C}$ are the roots of $f(s)$ (i.e., the poles of $Y(s)$ ). Note that above we assumed that $f(s)$ has no multiple roots (i.e., $\alpha_{j} \neq \alpha_{k}$ for $j \neq k$ ), which is valid for a general choice of $m, E_{0}, E_{i}$, and $\tau_{i}$. From the Heaviside cover-up method, the coefficients $C_{j} \in \mathbb{C}$ are determined as

$$
C_{j}=\lim _{\xi \rightarrow \alpha_{j}} \frac{\xi-\alpha_{j}}{\xi f(\xi)} .
$$

Applying the inverse Laplace transform to Eq. (A4), we obtain the temporal development of strain Eq. (6), where $\alpha_{j}$ is replaced with $-1 / \hat{\tau}_{j}$ to clarify the physical meaning.

The numerical analysis to obtain Fig. 4(a) was performed using an experimental relaxation function of an NBR compound [10] fitted with $N=25$. (see Appendix $\mathrm{C}$ for the referred stress relaxation function and the list of fitted parameters).

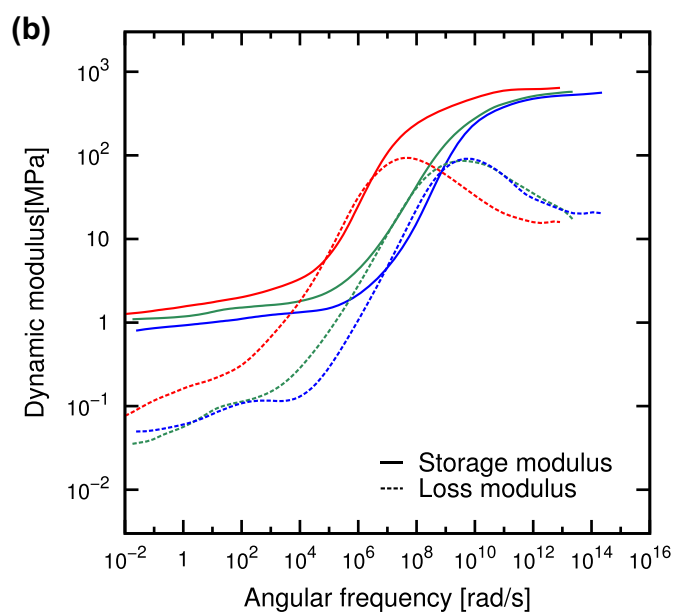

FIG. 10. Mechanical properties of NBR, SBR and BR with equal $c_{x}$ obtained by experiment. (a) Nominal stress-strain relationships under quasistatic condition. (b) Dynamic moduli; storage modulus (solid line) and loss modulus (dashed line). The entire curves of dynamic moduli were reconstructed by the time-temperature superposition from the experimental data at various temperature (see text). The data of dynamic moduli were smoothened for clarity. 

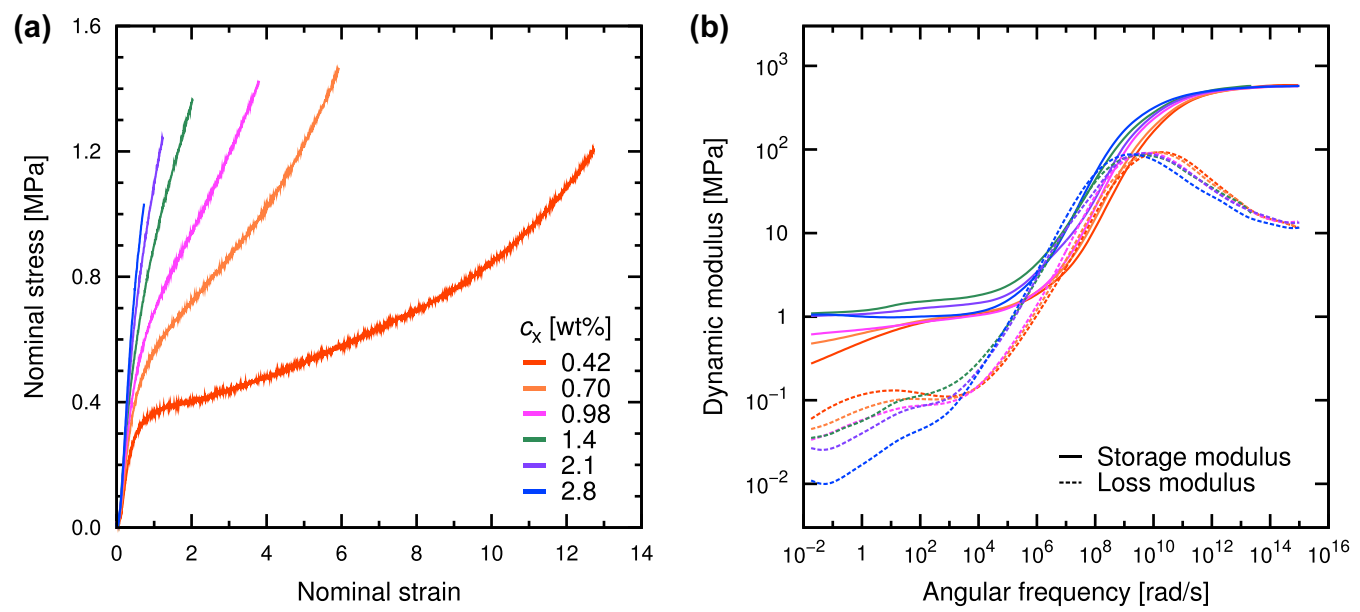

FIG. 11. Mechanical properties of SBR's with various $c_{x}$ obtained by experiment. (a) Nominal stress-strain relationships under quasistatic condition. (b) Dynamic moduli; storage modulus (solid line) and loss modulus (dashed line). The entire curves of dynamic moduli were reconstructed by the time-temperature superposition from the experimental data at various temperature (see text).

\section{APPENDIX B: MECHANICAL PROPERTIES OF RUBBER SAMPLES}

Figures 10 and 11 show the stress-strain relationships and the dynamic moduli of the unfilled rubber samples listed in Table I. The full range of dynamic moduli was reconstructed by applying the time-temperature superposition to the frequency sweep data from 0.5 to $50 \mathrm{~Hz}$ at various temperatures from 193 to $373 \mathrm{~K}$.

\section{APPENDIX C: MATERIAL PARAMETERS FOR STEP-LOADING MODEL}

We determine the material parameters in the Step-Loading Model (SLM) to obtain the results shown in Fig. 3(a). SLM with $N$ Maxwell segments has $2 N+2$ material parameters, i.e., $m, E_{0}, E_{i}$ and $\tau_{i}(i=1, \ldots, N)$.

The Prony-series parameters $E_{0}, E_{i}$ and $\tau_{i}$ were determined to reproduce an experimental stress relaxation function. We employed the stress relaxation function of acrylonitrilebutadiene rubber (NBR) with carbon black filler shown in Fig. 12, which is obtained by the previous experiment [10] and adopted in the precedent finite-element method analysis [20]. We fixed the relaxation times $\tau_{i}$ such that two $\tau_{i}$ 's are assigned for each digit of time within $t=10^{-10}-10^{2} \mathrm{~s}$; i.e., $N=25$. The discrete spectrum $E_{i}$ was optimized to reproduce the experimental stress relaxation function. Table II lists the obtained parameter set and it was confirmed in Fig. 12 that the Prony-series approximation well reproduces the experimental stress relaxation function.

The effective mass $m$ has the unit of mass per unit area and is dependent on the half specimen size along tension $L_{0} / 2$. Thus we estimated $m$ as

$$
m \gtrsim \frac{\rho L_{0}}{2},
$$

where $\rho$ denotes the mass density. In addition, the shear term in the minimal model for crack propagation (MMCP) can contribute to the effective mass because the effect of the shear term is proportional to $\frac{\partial^{2} \varepsilon}{\partial t^{2}}$ at the steady state (see Eq. (5) in Ref. [12] with $\frac{\partial}{\partial t}=V \frac{\partial}{\partial x}$ ). For the present numerical analysis we employed $m=3 \rho L_{0}$. Here we chose $\rho=1.0 \mathrm{~g} / \mathrm{cm}^{3}$, a typical value of regular polymer solids.

\section{APPENDIX D: DERIVATION OF THE ACCORDANCE OF TIME CONSTANTS BETWEEN SLM AND MMCP}

To show Eqs. (9) and (10), we consider SLM with a single time constant $\tau:=\tau_{1}$ (i.e., Eq. (3) with $N=1: E(t)=E_{0}+$ $\left.E_{1} e^{-t / \tau}\right)$, and prove the inequalities given as

$$
\begin{gathered}
-\frac{1}{(\lambda-1) \tau}<\alpha_{1}<-\frac{1}{\lambda \tau}, \\
-\frac{1}{2 \tau}\left(1-\frac{1}{\lambda}\right)<\operatorname{Re} \alpha_{2}<-\frac{1}{2 \tau}\left(1-\frac{1}{\lambda-1}\right),
\end{gathered}
$$

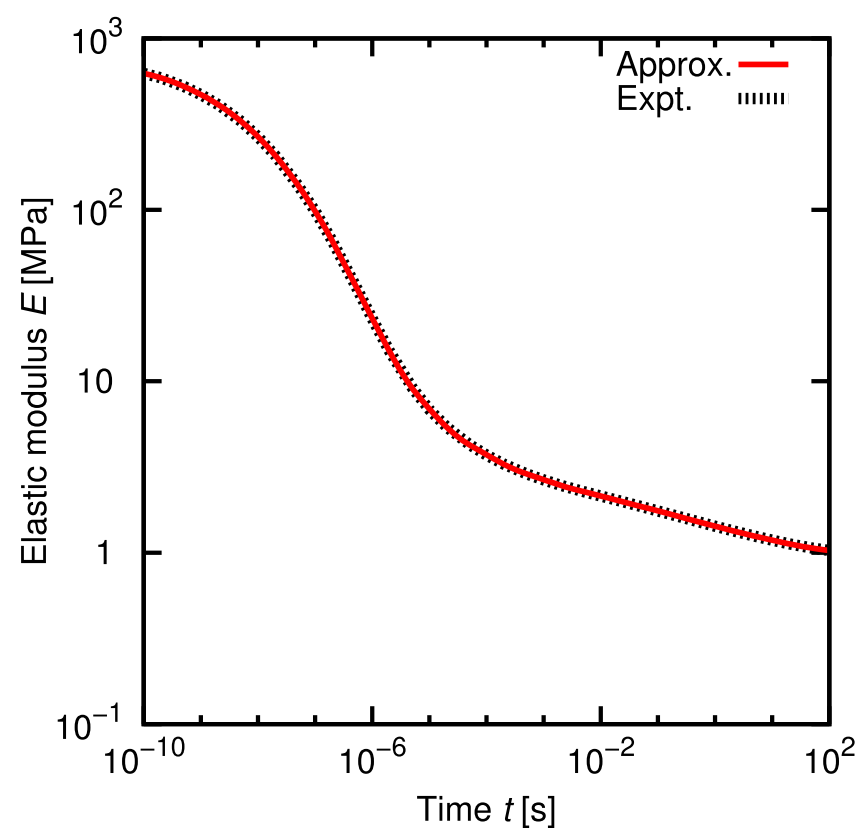

FIG. 12. Stress relaxation function of filled NBR by experiment [10] (dashed black line) and its Prony-series approximation (solid red line) employed for SLM analysis. 
TABLE II. Material parameters of SLM based on the previous experiment [10]. The index $i=0$ is assigned for the relaxed modulus $\left(\tau_{0}=\infty\right)$.

\begin{tabular}{|c|c|c|c|c|c|}
\hline$i$ & $\tau_{i}(\mathrm{~s})$ & $E_{i}(\mathrm{MPa})$ & $i$ & $\tau_{i}(\mathrm{~s})$ & $E_{i}(\mathrm{MPa})$ \\
\hline 0 & $(\infty)$ & 0.994 & 13 & $1 \times 10^{-4}$ & 1.33 \\
\hline 1 & $1 \times 10^{-10}$ & 27.8 & 14 & $3 \times 10^{-4}$ & 0.272 \\
\hline 2 & $3 \times 10^{-10}$ & 84.5 & 15 & $1 \times 10^{-3}$ & 0.536 \\
\hline 3 & $1 \times 10^{-9}$ & 85.3 & 16 & $3 \times 10^{-3}$ & 0.158 \\
\hline 4 & $3 \times 10^{-9}$ & 108 & 17 & $1 \times 10^{-2}$ & 0.271 \\
\hline 5 & $1 \times 10^{-8}$ & 118 & 18 & $3 \times 10^{-2}$ & 0.165 \\
\hline 6 & $3 \times 10^{-8}$ & 97.6 & 19 & $1 \times 10^{-1}$ & 0.204 \\
\hline 7 & $1 \times 10^{-7}$ & 79.3 & 20 & $3 \times 10^{-1}$ & 0.164 \\
\hline 8 & $3 \times 10^{-7}$ & 41.6 & 21 & 1 & 0.168 \\
\hline 9 & $1 \times 10^{-6}$ & 22.7 & 22 & 3 & 0.117 \\
\hline 10 & $3 \times 10^{-6}$ & 5.77 & 23 & $1 \times 10^{1}$ & 0.131 \\
\hline 11 & $1 \times 10^{-5}$ & 4.27 & 24 & $3 \times 10^{1}$ & 0.0767 \\
\hline 12 & $3 \times 10^{-5}$ & 0.806 & 25 & $1 \times 10^{2}$ & 0.0903 \\
\hline
\end{tabular}

when the effective mass $m$ is sufficiently small:

$$
m \leqslant 3 \tau^{2} E_{0} \quad \text { and } \quad \lambda>2 .
$$

Here, $\alpha_{i}$ are the roots of $f(s)=0$ with the characteristic equation for the strain response, i.e., Eq. (A3) with $N=1$ :

$$
f(s)=m s^{2}+E_{0}+\frac{E_{1} s}{s+1 / \tau} .
$$

Since $\lambda$ is sufficiently large $\left(\lambda \simeq 10^{3}\right)$ for typical elastomers, the inequalities (D1) and (D2) yield the approximate expressions $\alpha_{1} \simeq-1 /(\lambda \tau)$ and $\operatorname{Re} \alpha_{2} \simeq-1 /(2 \tau)$. By using the definitions, $\hat{\tau}_{\text {slow }}:=\hat{\tau}_{1}, 1 / \hat{\tau}_{\text {fast }}:=\operatorname{Re}\left(1 / \hat{\tau}_{2}\right)$, and $\alpha_{i}=-1 / \hat{\tau}_{i}$, the above expressions mean Eqs. (9) and (10).

To evaluate the roots of $f(s)=0$, we multiply the equality $f(s)=0$ by $(s \tau+1) / E_{0}$ and obtain the characteristic equation $g(s)=0$ with

$$
g(s):=\frac{m \tau}{E_{0}} s^{3}+\frac{m}{E_{0}} s^{2}+\tau \lambda s+1,
$$

where we use $\lambda:=1+E_{1} / E_{0}$. Note that the roots of $f(s)=$ 0 are equivalent with those of $g(s)=0$, because $f(-1 / \tau) \neq$ 0 . First, we show if the positive number (effective mass) $m$ is sufficiently small, then $g(s)=0$ has one negative root $\alpha_{1}$ in the range $-1 / \tau<\alpha_{1}<-1 /(\lambda \tau)$ and two complex roots $\alpha_{2}, \alpha_{3}$. To show this statement, we evaluate the function $g(s)$ on the real axis. The derivative of $g(s)$ is

$$
g^{\prime}(s)=\frac{3 m \tau}{E_{0}}\left(s+\frac{1}{3 \tau}\right)^{2}+\tau \lambda-\frac{m}{3 \tau E_{0}} .
$$

If $\tau \lambda-m /\left(3 \tau E_{0}\right)>0$, i.e., $m \leqslant 3 \tau^{2} E_{0}$, then $g^{\prime}(s) \geqslant 0$, (which has equality at most one point) and $g(s)$ is strictly increasing for arbitrary real number $s$. Since $\lambda>1$, we have

$$
g\left(-\frac{1}{\lambda \tau}\right)=\frac{m(\lambda-1)}{\lambda^{3} \tau^{2} E_{0}}>0
$$

and

$$
g\left(-\frac{1}{\tau}\right)=1-\lambda<0
$$

Therefore $g(s)=0$ has one negative root $\alpha_{1}$ in the range $-1 / \tau<\alpha_{1}<-1 /(\lambda \tau)$. Since $g(s)=0$ is a cubic equation with real coefficients, the remaining two complex (nonreal) roots $\alpha_{2}, \alpha_{3}$ are complex conjugates of each other. Second, we show inequality (D1) when the conditions (D3) hold. We have

$$
\begin{aligned}
g\left(-\frac{1}{(\lambda-1) \tau}\right) & =\frac{1}{\lambda-1}\left[\frac{m(\lambda-2)}{\tau^{2} E_{0}(\lambda-1)^{2}}-1\right] \\
& \leqslant \frac{1}{\lambda-1}\left[\frac{3(\lambda-2)}{(\lambda-1)^{2}}-1\right] \\
& =\frac{-1}{(\lambda-1)^{3}}\left[\left(\lambda-\frac{5}{2}\right)^{2}+\frac{3}{4}\right]<0 .
\end{aligned}
$$

Therefore $\alpha_{1}$ satisfies inequality (D1). Finally, we show inequality (D2). According to Vieta's formulas (formulas that relate the coefficients of a polynomial to sums and products of its roots), the characteristic equation $g(s)=0$ gives $\alpha_{1}+$ $\alpha_{2}+\alpha_{3}=-1 / \tau$. This relation is rewritten as $\alpha_{1}=-1 / \tau-$ $2 \operatorname{Re} \alpha_{2}$. Substituting this relation into inequality (D1), we have

$$
-\frac{1}{(\lambda-1) \tau}<-\frac{1}{\tau}-2 \operatorname{Re} \alpha_{2}<-\frac{1}{\lambda \tau} \text {. }
$$

Therefore we have inequality (D2).

\section{APPENDIX E: ESTIMATION OF EFFECTIVE ELASTIC MODULUS IN SLM}

We show the correspondence between the slow/fastvelocity regimes in SLM and the rubbery/glassy states in the case of stress relaxation functions $E(t)$ with general $N$. We numerically evaluate the temporal development of the effective elastic modulus $E_{\mathrm{eff}}(t):=\sigma^{\mathrm{ext}} / \varepsilon(t)$ and compare it to $E(t)$.

We consider the following two cases, i.e., $m=0$ and $m>$ 0 . Figure 13 shows $E_{\text {eff }}(t)$ for $m=0$ and $m>0$ compared to $E(t)$. In both cases, the long-term behavior of $E_{\text {eff }}(t)$ is common and $E_{\text {eff }}(t)$ converges to the stress relaxation function $E(t)$. On the other hand, the short-term behavior is affected by 


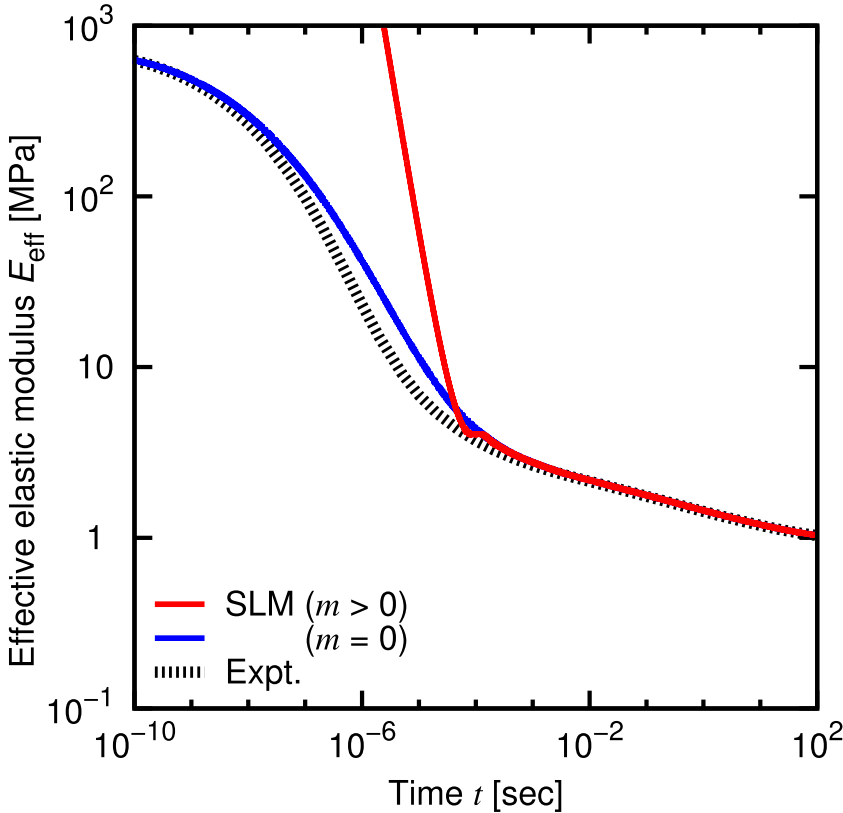

FIG. 13. Effective elastic moduli $E_{\text {eff }}(t)$ in the cases of $m=0$ and $m>0$, compared with the original stress relaxation function $E(t)$ obtained by an experiment [10].

the mass term. For $m=0$, the short-term behavior is directly related to $E(t)$ as well as the case of the long-term behavior. Only slight discrepancy is found between $E_{\text {eff }}(t)$ and $E(t)$ at the viscous-state region. For $m>0$, the mass-term effect



FIG. 14. Effective elastic modulus $E_{\text {eff }}(t)$ compared to the original stress relaxation function $E(t)$ for a massless Zener element.

is dominant on the short-term behavior, resulting in a harder response.

As mentioned above, the slight discrepancy between $E(t)$ and $E_{\text {eff }}(t)$ was found and is mainly due to the viscous behavior; its effect can be analytically shown with a massless Zener element. For a massless Zener element with the stress relaxation function $E(t)=E_{1} e^{-t / \tau}+E_{0}$, the effective elastic modulus under a stepwise load $E_{\text {eff }}(t)$ is evaluated from Eq. (6) as $E_{\mathrm{eff}}(t)=E_{1} e^{-t /(\lambda \tau)}+E_{0}$. Comparing $E_{\mathrm{eff}}(t)$ to $E(t)$, we find a retardation of mechanical response by the factor of $\lambda$ at the viscous-state region, as shown in Fig. 14
[1] S. Beurrot, B. Huneau, and E. Verron, In situ SEM study of fatigue crack growth mechanism in carbon black-filled natural rubber, J. Appl. Polym. Sci. 117, 1260 (2010).

[2] A. Livne, O. Ben-David, and J. Fineberg, Oscillations in Rapid Fracture, Phys. Rev. Lett. 98, 124301 (2007).

[3] C.-H. Chen, E. Bouchbinder, and A. Karma, Instability in dynamic fracture and the failure of the classical theory of cracks, Nat. Phys. 13, 1186 (2017).

[4] W. Wang and S. Chen, Hyperelasticity, Viscoelasticity, and Nonlocal Elasticity Govern Dynamic Fracture in Rubber, Phys. Rev. Lett. 95, 144301 (2005).

[5] P. J. Petersan, R. D. Deegan, M. Marder, and H. L. Swinney, Cracks in Rubber Under Tension Exceed the Shear Wave Speed, Phys. Rev. Lett. 93, 015504 (2004).

[6] M. Marder, Shock-Wave Theory for Rupture of Rubber, Phys. Rev. Lett. 94, 048001 (2005).

[7] M. Marder, Supersonic rupture of rubber, J. Mech. Phys. Solids 54, 491 (2006).

[8] A. Kadir and A. G. Thomas, Tear behavior of rubbers over a wide range of rates, Rubber Chem. Technol. 54, 15 (1981).

[9] K. Tsunoda, J. J. C. Busfield, C. K. L. Davies, and A. G. Thomas, Effect of materials variables on the tear behaviour of a non-crystallising elastomer, J. Mater. Sci. 35, 5187 (2000).

[10] Y. Morishita, K. Tsunoda, and K. Urayama, Velocity transition in the crack growth dynamics of filled elastomers: Contributions of nonlinear viscoelasticity, Phys. Rev. E 93, 043001 (2016).
[11] Y. Morishita, K. Tsunoda, and K. Urayama, Crack-tip shape in the crack-growth rate transition of filled elastomers, Polymer 108, 230 (2017).

[12] N. Sakumichi and K. Okumura, Exactly solvable model for a velocity jump observed in crack propagation in viscoelastic solids, Sci. Rep. 7, 8065 (2017).

[13] See Supplemental Materials at http://link.aps.org/supplemental/ 10.1103/PhysRevMaterials.5.073608 [SupplMovie1Slow.wmv] for the slow crack propagation and [SupplMovie2-Fast.wmv] for the fast crack propagation, respectively.

[14] H. W. Greensmith and A. G. Thomas, Rupture of rubber. III. Determination of tear properties, J. Polym. Sci. 18, 189 (1955).

[15] H. W. Greensmith, Rupture of rubber. IV. Tear properties of vulcanizates containing carbon black, J. Polym. Sci. 21, 175 (1956).

[16] G. Carbone and B. N. J. Persson, Crack motion in viscoelastic solids: The role of the flash temperature, Eur. Phys. J. E 17, 261 (2005).

[17] G. Carbone and B. N. J. Persson, Hot Cracks in Rubber: Origin of the Giant Toughness of rubBerlike Materials, Phys. Rev. Lett. 95, 114301 (2005).

[18] F. D'Amico, G. Carbone, M. M. Foglia, and U. Galietti, Moving cracks in viscoelastic materials: Temperature and energyrelease-rate measurements, Eng. Fract. Mech. 98, 315 (2013).

[19] W. G. Knauss, A review of fracture in viscoelastic materials, Int. J. Fract. 196, 99 (2015). 
[20] A. Kubo and Y. Umeno, Velocity mode transition of dynamic crack propagation in hyperviscoelastic materials: A continuum model study, Sci. Rep. 7, 42305 (2017).

[21] K. Grosch, J. A. C. Harwood, and A. R. Payne, Breaking energy of rubbers, Nature 212, 497 (1966).

[22] K. A. Grosch, J. A. C. Harwood, and A. R. Payne, Hysteresis in polymers and its relation to strength, Rubber Chem. Technol. 41, 1157 (1968).

[23] J. A. C. Harwood and A. R. Payne, Hysteresis and strength of rubbers, J. Appl. Polym. Sci. 12, 889 (1968).

[24] T. Horst, K. Reincke, S. Ilisch, G. Heinrich, and W. Grellmann, Fracture surface statistics of filled elastomers, Phys. Rev. E 80, 046120 (2009).

[25] E. Bouchbinder, A. Livne, and J. Fineberg, Weakly Nonlinear Theory of Dynamic Fracture, Phys. Rev. Lett. 101, 264302 (2008).

[26] E. Bouchbinder, A. Livne, and J. Fineberg, The 1/r singularity in weakly nonlinear fracture mechanics, J. Mech. Phys. Solids 57, 1568 (2009).
[27] T. Goldman Boué, R. Harpaz, J. Fineberg, and E. Bouchbinder, Failing softly: a fracture theory of highly-deformable materials, Soft Matter 11, 3812 (2015).

[28] A. Livne, E. Bouchbinder, I. Svetlizky, and J. Fineberg, The near-tip fields of fast cracks, Science 327, 1359 (2010).

[29] T. Tomizawa and K. Okumura, Velocity jump in the crack propagation induced on a semi-crystalline polymer sheet by constant-speed stretching, Polymer 173, 166 (2019).

[30] Y. Morishita, K. Tsunoda, and K. Urayama, Universal relation between crack-growth dynamics and viscoelasticity in glassrubber transition for filled elastomers, Polymer 179, 121651 (2019).

[31] J. Murai, T. Nakajima, T. Matsuda, K. Tsunoda, T. Nonoyama, T. Kurokawa, and J. P. Gong, Tough double network elastomers reinforced by the amorphous cellulose network, Polymer 178, 121686 (2019).

[32] C. Liu, H. Kadono, H. Yokoyama, K. Mayumi, and K. Ito, Crack propagation resistance of slide-ring gels, Polymer 181, 121782 (2019). 